\title{
COVID-19: The Impact of Social Isolation on Mood, Behavior and Biological Rhythms
}

\author{
Pritakshi Das ${ }^{1}$, Priya Ranjan Avinash ${ }^{2}$, Vrinda Saxena ${ }^{3}$, Jitendra Kumar Sinha ${ }^{4}$ \\ ${ }^{1}$ Amity University, Noida \\ ${ }^{2}$ Associate Professor, Department of Psychiatry, Swami Rama Himalayan University Dehradun, \\ Uttarakhand \\ ${ }^{3}$ Swami Rama Himalayan University, Dehradun \\ ${ }^{4}$ Amity University, Noida
}

Corresponding author: Priya Ranjan Avinash

Email -drpriyaranjan.avinash@gmail.com

\begin{abstract}
Background: A new strain of Corona virus, SARS- CoV-2, emerged in the Wuhan city of China in December 2019. Due to fast and easy transmission of this respiratory virus the world was under a lockdown for a long time. The purpose of this study was to find out changes in behavior, mood and biological rhythm of people as a result of social distancing and isolation during the COVID-19 pandemic.

Methodology: The study was conducted in the months of April and May 2020. This was the time when the lockdown was at its peak. It used a self-rating web based structured questionnaire formulated on Google forms which was circulated through different platforms for people to fill after giving their consents.

Results: Highest numbers of respondents were from India (30.2\%), USA (21.5\%) and UK (11.5\%). Our results showed that $46.3 \%$ subjects experienced an increase in problems related to sleep. Even though they were getting sufficient amounts of sleep, it was unstructured, and the quality of sleep was affected. The appetite was seen to be good with $68.2 \%$ reporting no changes. There was an increase in substance use in $43.8 \%$ of the participants while in the lockdown. Our study revealed that $34.2 \%$ participants had anxiety and $15.2 \%$ showed depressive symptoms. More than $50 \%$ respondent's perceived lack of motivation, lack of energy, nervousness and irritability, and $13.4 \%$ reported having suicidal thoughts regularly.

Conclusions: This data shows that social isolation due to the COVID-19 lockdown has caused significant changes in the mood, behavior and biological rhythm of people.
\end{abstract}

Keywords: mood, sleep, behavior, biological rhythm, COVID-19

(Paper received $-12^{\text {th }}$ December 2020, Peer review completed $-5^{\text {th }}$ January 2021)

(Accepted $-8^{\text {th }}$ January 2021)

\section{INTRODUCTION}

In December of 2019, several patients were hospitalized in Wuhan province of China having common symptoms, but no known pathogen could be identified. The samples were then sent for etiological purposes and were identified as Severe Acute Respiratory Syndrome Coronavirus 2 (SARS- CoV-2) [1]. COVID-19 is a pandemic that has stirred the world. SARS-CoV-2, the causing agent of this disease, is a highly contagious virus and causes respiratory illnesses in people. This has put a massive burden on the healthcare systems around the world. All the nations are trying to flatten the curve, and in an attempt in achieving this goal, have implemented large scale lockdowns. The immune system of people plays a pivotal role in the disease; individuals suffering from chronic co-morbid illnesses get the most complications from this disease [1-2]. Elderly men with long term illness or ARDS showed the highest rate of deaths with the highest recoveries falling in the 24 to 55 age group [3-4]. From the past literature on similar pandemics, it can be 
said that the most vulnerable groups consist of children and adolescents, elderly people, minority groups, females, those from lower socio-economic groups, and people with pre-existing mental health conditions [5]. WHO reports that the primary psychological effect as a result of this pandemic has been elevated rates of anxiety and stress? Businesses have been affected; people are losing their jobs, their apartments, having difficulties in getting even the basic necessities. Domestic confinement has also resulted in increase in isues within household like domestic violence and adjustment issues. These factors contribute to deteriorating psychological health of population. During such adverse and uncertain times, the need for social support is the highest, but this forced solitude has severed social connectedness and may take a considerable toll on an individual's mental health [6]. In a study conducted post the SARS epidemic, the subjects reported feeling bored, isolated, frustrated, annoyed, and worried while in quarantine [7]. Levels of loneliness, depression, frustration and substance use are most likely to rise too. Social isolation has been known to affect biological rhythms as well. Timely mental health care must be provided to people, especially those in the vulnerable groups so that the psychological well-being of the population can be maintained [8]. The aim of our study was to find out the impact of social isolation on behavior, mood and biological rhythm of people due to lockdown during the COVID-19 pandemic.

\section{METHODOLOGY}

\section{Design and Data collection}

This was a cross-sectional study with continuous sampling conducted online using a self-rating web based questionnaire, formulated on Google forms. The questionnaire developed covered various aspects of a person's life and functioning like demographics, pre-existing illnesses, problems faced as a result of lockdown, substance use, sleep habits, appetite, bowel movements, and changes in behavior, mood and biological rhythmicity. The questionnaire was circulated through different platforms. Subjects were asked to fill questionnaire after giving a valid consent.

\section{Statistical analysis}

Data collected was analysed using Google forms inbuilt statistical tools. Mean, median, mode, standard deviation frequency table, and bar diagrams were prepared.

\section{RESULTS}

\section{Demographic information of the participants}

In our study, 679 participants from 71 countries took part over the span of a month (April 15, 2020 - May $15,2020)$. Maximum respondents were from India $(n=205)$, The United States of America $(n=146)$ and The United Kingdom $(n=78)$. These are some of the most heavily affected countries in the World. The majority of the participants were female (76.1\%), followed by males $(23.3 \%)$ and other genders $(0.4 \%)$. Most of the respondents belonged to the middle-income group (89.6\%). More than $50 \%$ were in the age group of $15-30$ years. Majority of the respondents were single (57.3\%). Lockdown can be especially difficult for people who are living alone, $35.1 \%$ reported not isolating with their families during the lockdown. In terms of education, majority had a bachelor's degree and higher (74.4\%) (Table 1)

The respondents suffered various inconveniences due to the lockdown. We see that, $24.5 \%$ reported suffering from financial loss, $17.1 \%$ had difficulty in getting essential items, $9.3 \%$ of the subjects reported unavailability of medicines, $20.4 \%$ reported having difficulty in accessing healthcare facilities and $18.9 \%$ suffered inconvenience due to the absence of their house help (Figure 1). 
Table 1: Socio-demographic details of the participants

\begin{tabular}{|c|c|c|}
\hline Variables & Frequency & Percentage (\%) \\
\hline \multicolumn{3}{|l|}{ Gender } \\
\hline Female & 517 & 76.1 \\
\hline Male & 158 & 23.3 \\
\hline Other & 4 & 0.4 \\
\hline \multicolumn{3}{|l|}{ Age } \\
\hline $0-15$ years & 2 & 0.3 \\
\hline $15-30$ years & 384 & 56.5 \\
\hline $31-45$ years & 194 & 28.6 \\
\hline More than 45 years & 97 & 14.3 \\
\hline Prefer not to answer & 2 & 0.3 \\
\hline \multicolumn{3}{|l|}{ Education } \\
\hline Graduation & 256 & 37.7 \\
\hline Post-graduation & 249 & 36.7 \\
\hline Intermediate/Diploma & 95 & 14 \\
\hline High school certificate & 74 & 10.9 \\
\hline Middle school certificate & 4 & 0.6 \\
\hline Primary school certificate & 1 & 0.1 \\
\hline \multicolumn{3}{|l|}{ Marital status } \\
\hline Single & 389 & 57.3 \\
\hline Married & 166 & 24.4 \\
\hline Living with a partner & 85 & 12.5 \\
\hline Divorced & 36 & 5.3 \\
\hline Widowed & 3 & 0.5 \\
\hline \multicolumn{3}{|l|}{ Living with family } \\
\hline Yes & 441 & 64.9 \\
\hline No & 238 & 35.1 \\
\hline \multicolumn{3}{|l|}{ Income Class } \\
\hline Middle Class & 608 & 89.6 \\
\hline Upper Class & 41 & 6.0 \\
\hline Lower Class & 30 & 4.4 \\
\hline
\end{tabular}

Figure 1: Problems faced by individuals during the lockdown

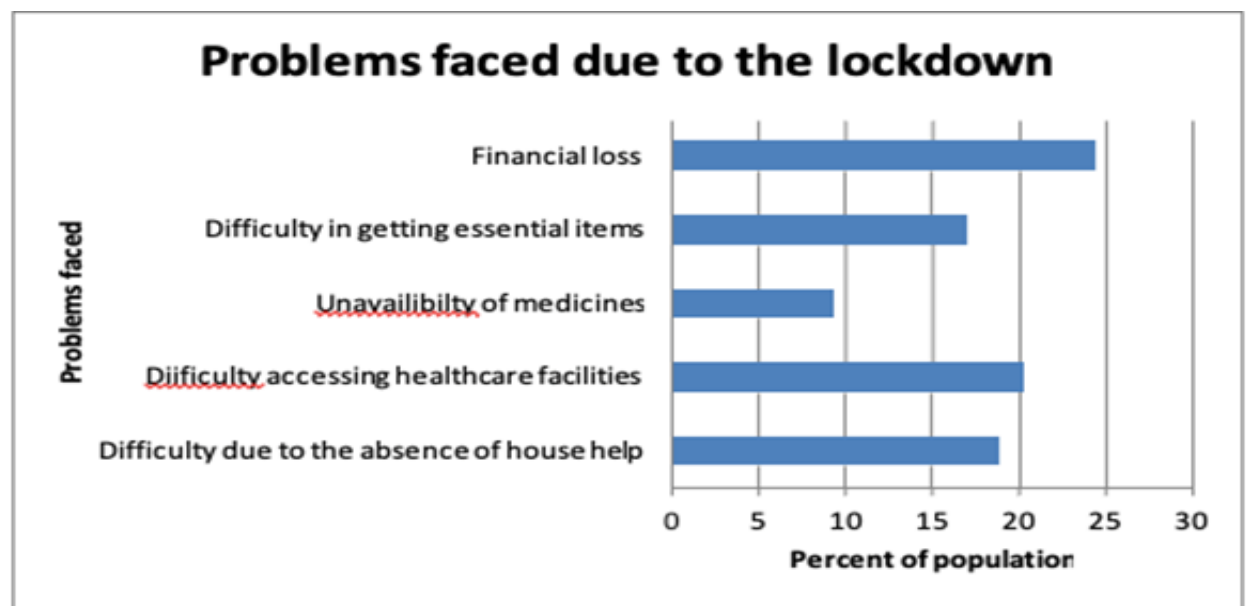




\section{Substance usage}

$70.8 \%$ of the respondents said that they have consumed some form of substance at least once in their lifetime. Out of them, $57.8 \%$ use it on regular basis (at least once or twice in a week). Majority of the participants who indulged in substance use on a regular basis consisted of people who consumed spirits (71\%), followed by beer drinkers (56.9\%), cigarette smokers (42.8\%), marijuana consumers (34.9\%) and MDMA users $(12.5 \%)$. Small number of people also reported using cocaine, LSD, codeine etc.

\section{Frequency of substance use}

Out of the $71 \%$ of regular users, $43.8 \%$ reported an increase in their usage, $33.9 \%$ said their consumption has decreased and $22.3 \%$ said that there has been no change in their usage as compared to pre-lockdown period.

\section{Sleep Conditions monitored to assess changes in sleep habits of the participants}

On average, the subjects were getting 8 hours of sleep every day. $61.14 \%$ of respondents reported going to bed after midnight, and out of them $36.2 \%$ went to bed after $5 \mathrm{am}$. They reported experiencing changes in their sleeping patterns and various other sleep disturbances during the lockdown. The factors recorded were difficulty in falling asleep (47.2\%), persistent thoughts before bed (48.8\%), difficulty in relaxing before bed (46.4\%), feeling lethargic after a full night's sleep (54.8\%), waking up in too early and being unable to fall asleep (34.7\%). The participants said that they experienced it more than half number of days in a week.

Studies have shown that traumatic events (such as a pandemic) can result in frequent nightmares or vivid mental imagery in dreams. We see that $44.5 \%$ did not experience any such nightmares, $28.9 \%$ reported occasionally having them, $13.1 \%$ were having these dreams more than half number of days in a week, $6.6 \%$ had them almost every day and $6.8 \%$ had them regularly.

\section{Appetite}

Appetite of the participants were monitored by several factors which included desire to eat, number of meals in a day, satiety after meals and whether or not they felt nauseated after meals. $33.9 \%$ of the participants had an average appetite, followed by $31.6 \%$ who had a good appetite, $21.5 \%$ had a very good appetite, $9.9 \%$ of them had a poor appetite and $3.1 \%$ of them had a very poor appetite.

\section{Changes in sleep and appetite as compared to pre-lockdown period}

A graphical representation of the changes experienced by the participants in their sleep cycle and eating habits as compared to pre-lockdown period. $67.3 \%$ of the respondents reported experiencing the issues (difficulty in falling asleep, persistent thoughts before bed etc.) prior to the lockdown as well and $34.7 \%$ said that they haven't experienced any such things before. We see that out of the $67.3 \%$ participants who did experience the symptoms before the lockdown, $46.3 \%$ said that there was an increase in frequency as compared to the pre- lockdown period, $6.6 \%$ said that the frequency has decreased and for $47.1 \%$ respondents the frequency remained the same. $28.3 \%$ reported an increase, $3.6 \%$ reported a decrease in the frequency and $68.3 \%$ reported no change in their appetite as compared to the pre-lockdown period.

\section{Bowel Movement}

Colonic motility follows the circadian clock as well. Gastrointestinal problems such as diarrhea and constipation are widespread among shift-workers and frequent time-zone travelers, both of these situations are known to disrupt in body's internal clock. Disruptions in circadian rhythms have also been associated with irritable bowel syndrome (IBS) flare-ups, which can cause diarrhea, constipation, senses of incomplete defecation etc. We see that $18.3 \%$ of the participants had diarrhea or loose/watery stool more than half number of days in a week, $25.5 \%$ reported feeling constipated and $29.5 \%$ said they had sense of incomplete evacuation after defecation.

\section{Mood and behavioral changes}

The study monitored various factors to understand the changes in mood and behavior of the participants. The participants reported getting easily irritated (50.5\%), feeling agitated (42.1\%), obsessive thinking (47.5\%), feeling of nervousness without any apparent reason (51.8\%), difficulty concentrating (46.\%), lack of energy 
(54.3\%), decreased interest in things that were pleasurable before (43.6\%), lack of motivation (57.1\%), feeling of failure (43.4\%), feeling a lack of purpose (33.9\%) and suicidal thoughts (13.4\%) (Fig. 8).

\section{Frequency of changes in mood and behavior as compared to before the lockdown.}

Around $40 \%$ of the respondents experienced these symptoms for the first time on frequent basis. $59.7 \%$ of the subjects said that they had experienced these symptoms before the lockdown as well, among them, $47.6 \%$ reported an increase, $6.8 \%$ reported a decrease and $45.6 \%$ said there had been no changes as compared to before the lockdown.

\section{DISCUSSION}

This cross-sectional study describes the changes in biological rhythm and psychology due to social isolation during the COVID-19 lockdown. Pandemics, such as COVID-19, have been known to cause psychological distress and affect the mental health of people [9]. It is known that long term isolation can cause symptoms related to depression and anxiety [10]. Both of which have been known to frequently co-exist in a person [11]. The time spent quarantined is directly proportional to poorer mental health outcomes [12]. The effects may include mood disorders, anxiety, fear and panic, depression-like symptoms, stress disorders, insomnia, irritability, loneliness, dejection, frustration, and boredom. Participants in our study reported feeling irritated (50.5\%); they felt agitated (42.1\%), had difficulty concentrating (51.8\%), felt nervous (51.8\%) and indulged in obsessive thinking (47.5\%). They also reported a lessened interest in doing things that were pleasurable to them before the lockdown (43.6\%), lacked motivation to work (57.1\%), and had feeling of failure (43.4\%), lack of purpose (33.9\%) and suicidal thoughts (13.4\%). Social isolation and loneliness has been known to be one of the major risk factors for suicidal tendencies [13]. Anxiety and depression during the lockdown can result in an increase in such tendencies, especially for the vulnerable groups. A myriad of factors such as unemployment, poverty and stress related psychiatric conditions such as mood disorders and substance use disorders can give impetus to such tendencies [14-15]. The lockdown has been especially hard for patients battling substance use disorders (SUDs). It has been established that co-morbidities increase the risk of complications. People who smoke have compromised lung functioning, and opioid use can lead to hypoxia, these individuals thus have a higher risk of serious outcomes from this disease as compared to the general population [16]. As most of the liquor stores have been closed due to the lock-down, individuals suffering from alcohol addiction may suffer due to forced abstinence which may cause serious health complications. Due to COVID-19 and associated lockdown, treatment centers have been closed and patients have been discharged without the completion of their treatment, this may lead to relapse [17]. Withdrawal from longterm cannabis use can cause irritability, anger, aggression, nervousness, anxiety, sleep disorders like insomnia, decreased appetite or weight loss, restlessness, and depressed mood [18]. Not much literature is available on the impact of social isolation due to COVID-19 concerning patients suffering from substance use disorders. But it is said to be risk factor for anxiety and depression which can lead to increase in suicidal tendencies, especially for people who are socially isolating. According to our study $43.8 \%$ respondents had an increase substance use and $33.9 \%$ respondents reported decreased frequency of usage.

In a study conducted on the general public of China, the researchers reported COVID-19 having a moderate to severe impact on the psychological health of more than $50 \%$ of the people. In the same study, it was established that $6.5 \%$ of the population reported having depressive symptoms; $28.8 \%$ reported moderate to severe anxiety, and $8.1 \%$ reported moderate to severe stress levels [19]. Similarly, a study conducted on Iranian college students measuring their levels of anxiety during the lockdown reported that $30.6 \%$ of the subjects showed moderate to severe anxiety and $9.8 \%$ showed very severe levels of anxiety [20]. These results are similar to our findings where $34.2 \%$ respondents reported having anxiety on regular basis. A longitudinal study measuring anxiety and depression of Chinese individuals again reported levels of anxiety and depression to be $28.8 \%$ and $16.5 \%$ respectively [21]. In our study $15.2 \%$ participants showed depressive symptoms. Our study also showed that $40.3 \%$ subjects developed new onset psychological distress during the lockdown. Lack of supplies, poor communication, and monetary concerns just aggravate these issues $[6,12,22-24]$. Financial loss can become a big problem during pandemics and resulting lockdowns. In our 
study $24.8 \%$ of respondents faced financial difficulties. We also looked into access to essentials like grocery, medication, as well as, availability of domestic help during the lockdown. Medicines and grocery are one of the most essential requirements and in our study $17.1 \%$ of the subjects had difficulty in getting essential household items and 9.3\% faced unavailability of medicines. This could be due to the panic buying and hoarding of the said items during the peak of the pandemic, the time when this study was conducted. $20.4 \%$ reported having difficulty in accessing healthcare facilities and $18.8 \%$ suffered inconvenience due to the absence of their house help. This resulted in increased burden of household work and further added to distress, especially for women.

Biological rhythm refers to a person's internal clock; it regulates biological functions such as sleep- wake cycle, appetite, colonic motility etc. Stress caused due to the pandemic and loneliness due to social isolation can affect the biological clock. Stress has been known to cause impaired sleep and even insomnia. Sleep quality can also be affected due to various other reasons like substance withdrawal, loneliness, anxiety, fear and obsessive thoughts among other things. Our study revealed $61.4 \%$ of the participants went to bed after midnight and among them $36.2 \%$ went to be after $5 \mathrm{am}$. The population also had difficulty in falling asleep $(47.2 \%)$, persistent thoughts before bed $(48.8 \%)$, difficulty in relaxing before bed $(46.4 \%)$, lethargy and daytime tiredness (54.8\%), and reported waking up and unable to go back to bed (34.7\%). The participants were getting on an average 8 hours of sleep per day. So it can be inferred that even though people were getting adequate amount of sleep, it was unstructured and the sleep quality was poor. An Italian study revealed $57.1 \%$ of the participants having poor sleep quality which is similar to our findings [25]. Emotions play a crucial role in the dreaming process. During such adverse times, spreading of false information is prevalent and can cause unnecessary anxiety and panic in the population. Using social media as a source of information while isolating can increase cyberchondria and cause information overload. The information people gather from television, newspapers etc. can also cause fear of the disease and anxiety. This can be represented as symbolic imagery during dreams and can result in vivid dreams and even nightmares. After $9 / 11$, people experienced vivid dreams and remembered them longer, ongoing researches suggest that this pandemic might have a similar effect. In our study , $13.1 \%$ of people said they are experiencing nightmares more than half days in a week during the lockdown, $6.6 \%$ experienced them almost every alternate day and $6.8 \%$ said they get such dreams regularly. Furthermore, biological clock is responsible for daily food intake, it was seen that $87 \%$ of the respondents had average to good appetite during the quarantine. Among whom $68.2 \%$ reported no change in their appetite as compared to before the lockdown, $28.3 \%$ reported an increase and appetite of $3.6 \%$ decreased. Colonic motility follows the biological clock as well, gastrointestinal problems such as diarrhea and constipation are widespread among shift-workers and frequent time-zone travelers, both of these situations are known to disrupt in body's internal clock [26]. Disruptions in circadian rhythms have also been associated with irritable bowel syndrome (IBS) flare-ups, which can cause diarrhea, constipation, senses of incomplete defecation etc. In our study $18.3 \%$ of the participants had diarrhea or loose/watery stool more than half number of days in a week, $25.5 \%$ reported feeling constipated and $29.5 \%$ said they had sense of incomplete evacuation after defecation.

Finally, previous research shows that the mental health effects of social isolation continue to persist even after the quarantine period has ended. Post the 2002 SARS epidemic, a range of avoidance behavior such as reduced direct contact with people and crowds, less social interaction, avoiding enclosed and public places, not returning to work, was seen in the population [27]. Long-term behavioral changes like excessive hand washing were also reported. Health workers who had been quarantined reported adverse mental health outcomes, such as, post-traumatic stress disorder, and feelings of isolation, depression, anxiety, loneliness, and helplessness [6]. This study will help in understanding the emotional, behavioral and biological changes in the population, add to the existing knowledge, and will help in devising new strategies for dealing with the psychological effects of the pandemic. 


\section{REFERENCES}

1. Wang D, Hu B, Hu C, Zhu F, Liu X, Zhang J, Wang B, Xiang H, Cheng Z, Xiong Y, Zhao Y. Clinical characteristics of 138 hospitalized patients with 2019 novel coronavirus-infected pneumonia in Wuhan, China. JAMA 2020;323(11):1061-9.

2. Huang C, Wang Y, Li X, Ren L, Zhao J, Hu Y, Zhang L, Fan G, Xu J, Gu X, Cheng Z. Clinical features of patients infected with 2019 novel coronavirus in Wuhan, China. Lancet 2020;395(10223):497-506.

3. Wang C, Horby P, Hayden F, Gao G. A novel coronavirus outbreak of global health concern. Lancet 2020;395(10223):470-3.

4. Yang Y, Li W, Zhang Q, Zhang L, Cheung T, Xiang YT. Mental health services for older adults in China during the COVID-19 outbreak. Lancet Psychiatry 2020;7(4):e19.

5. Perrin P, McCabe O, Everly G, Links J. Preparing for an Influenza Pandemic: Mental Health Considerations. Prehosp Disaster Med 2009;24(3):223-30.

6. Hawryluck L, Gold W, Robinson S, Pogorski S, Galea S, Styra R. SARS Control and Psychological Effects of Quarantine, Toronto, Canada. Emerg Infect Dis 2004;10(7):1206-12.

7. Reynolds D, Garay J, Deamond S, Moran M, Gold W, Styra R. Understanding, compliance and psychological impact of the SARS quarantine experience. Epidemiol Infection 2007;136(7):997-1007.

8. Xiang Y, Yang Y, Li W, Zhang L, Zhang Q, Cheung T, Ng C. Timely mental health care for the 2019 novel coronavirus outbreak is urgently needed. The Lancet Psychiatry 2020;7(3):228-9.

9. Bao Y, Sun Y, Meng S, Shi J, Lu L. 2019-nCoV epidemic: address mental health care to empower society. The Lancet 2020;395(10224):e37-8.

10. Han R, Kim Y, Park E, Kim J, Ryu C, Kim H, Lee J, Pahk K, Shanyu C, Kim H, Back S, Kim H, Kim Y, Na H. Long-Term Isolation Elicits Depression and Anxiety-Related Behaviors by Reducing Oxytocin-Induced GABAergic Transmission in Central Amygdala. Front Mol Neurosci 2018;11.

11. Ionescu D, Niciu M, Mathews D, Richards E, Zarate C. Neurobiology of Anxious Depression: a review. Depress Anxiety 2013;30(4):374-85.

12. Brooks SK, Webster RK, Smith LE, Woodland L, Wessely S, Greenberg N, Rubin GJ. The psychological impact of quarantine and how to reduce it: rapid review of the evidence. Lancet 2020;395(10227):912-20.

13. Calati R, Ferrari C, Brittner M, Oasi O, Olié E, Carvalho A, Courtet P. Suicidal thoughts and behaviors and social isolation: A narrative review of the literature. J Affect Disord 2019;245:653-67.

14. Kawohl W, Nordt C. COVID-19, unemployment, and suicide. The Lancet Psychiatry 2020;7(5):389-90.

15. Sher L. The impact of the COVID-19 pandemic on suicide rates. QJM: An International Journal of Medicine. 2020;113(10):707-12.

16. Volkow ND, Blanco C. Research on substance use disorders during the COVID-19 pandemic. J Subst Abuse Treat 2021;129:108385.

17. Arya S, Gupta R. COVID-19 outbreak: Challenges for Addiction services in India. Asian J Psychiatry 2020;51: 102086.

18. Patel J, Marwaha R. Cannabis use disorder. StatPearls [Internet]. 2019 Jun 5.

19. Wang C, Pan R, Wan X, Tan Y, Xu L, Ho C, Ho R. Immediate Psychological Responses and Associated Factors during the Initial Stage of the 2019 Coronavirus Disease (COVID-19) Epidemic among the General Population in China. Int J Environ Res Pub Health 2020;17(5):1729.

20. Moghanibashi-Mansourieh A. Assessing the anxiety level of Iranian general population during COVID-19 outbreak. Asian J Psychiatry 2020;51:102076.

21. Wang C, Pan R, Wan X, Tan Y, Xu L, McIntyre R, Choo F, Tran B, Ho R, Sharma V, Ho C. A longitudinal study on the mental health of general population during the COVID-19 epidemic in China. Brain Behav Immun 2020;87:40-8.

22. Bai Y, Lin C, Lin C, Chen J, Chue C, Chou P. Survey of Stress Reactions Among Health Care Workers Involved with the SARS Outbreak. Psychiatr Serv 2004;55(9):1055-7.

23. Cava M, Fay K, Beanlands H, McCay E, Wignall R. The Experience of Quarantine for Individuals Affected by SARS in Toronto. Pub Health Nurs 2005;22(5):398-406.

24. Usher K, Bhullar N, Jackson D. Life in the pandemic: Social isolation and mental health. J Clin Nurs 2020;29(15-16):2756-7.

25. Casagrande M, Favieri F, Tambelli R, Forte G. The enemy who sealed the world: Effects quarantine due to the COVID-19 on sleep quality, anxiety, and psychological distress in the Italian population. Sleep Med 2020;75:12-20.

26. Hoogerwerf W. Role of clock genes in gastrointestinal motility. Am J Physiol Gastrointest Liver Physiol 2010;299(3):549-55. 
27. Farooq A, Laato S, Islam A. Impact of Online Information on Self-Isolation Intention During the COVID-19 Pandemic: Cross-Sectional Study. J Med Internet Res 2020;22(5):e19128

Acknowledgements - Nil.

Conflict of Interest - Nil

Funding - Nil 